\title{
Evolution of indeterminate hepatocellular nodules at Gd-EOB-DPTA-enhanced MRI in cirrhotic patients
}

\author{
Massimo Galia ${ }^{1}$ Francesco Agnello ${ }^{1} \cdot$ Gianvincenzo Sparacia $^{1} \cdot$ Domenica Matranga $^{2} \cdot$ Domenico Albano $^{1}$. \\ Massimo Midiri ${ }^{1} \cdot$ Roberto Lagalla $^{1}$
}

Received: 7 November 2017 / Accepted: 6 March 2018

(c) Italian Society of Medical Radiology 2018

\begin{abstract}
Purpose To retrospectively analyze the evolution of indeterminate hepatocellular nodules in cirrhotic patients on serial GdEOB-DPTA-enhanced MRI, and to identify predictors of HCC development.

Materials and methods This IRB approved study included 33 cirrhotic patients with 69 indeterminate hepatocellular nodules (mean diameter $1.1 \mathrm{~cm}$ ) at baseline Gd-EOB-DPTA-enhanced MRI and a Gd-EOB-DPTA-enhanced-MRI follow-up of at least 2 years. Two radiologists evaluated size and signal intensity of each nodule at baseline and follow-up. Age, cirrhosis etiology, and HCC history were recorded. Data were compared between nodules that became HCCs at follow-up (HCC) and those that did not (no-HCC).

Results On follow-up, 5/69 nodules became HCCs and 64/69 showed indeterminate characteristics. HCC history was more frequently found in HCCs than in no-HCCs. Age, sex, and cirrhosis etiology were not significantly different between HCCs and no-HCCs. HCCs had a significantly greater baseline diameter and increase in size than no-HCCs. Hepatobiliary phase hypointensity was significantly more common in HCCs than in no-HCCs. Multivariate regression analysis showed that increase in size (OR 10.48; sensitivity, $100 \%$; specificity, $81.2 \% ; p<0.001)$ and hepatobiliary phase hypointensity (OR 1.02; sensitivity, $100 \%$; specificity, $78.1 \%$; $p<0.001$ ) was associated with HCC development.

Conclusion Indeterminate hepatocellular nodules at Gd-EOB-DPTA-enhanced MRI in cirrhotic patients rarely became HCCs. Hepatobiliary phase hypointensity had a weak association with HCC development.
\end{abstract}

Keywords HCC $\cdot$ Indeterminate hepatocellular nodule $\cdot$ Cirrhosis $\cdot$ Gd-EOB-DTPA $\cdot$ Liver MRI

\section{Introduction}

Current diagnosis and management of hepatocellular carcinoma (HCC) are largely guided by imaging results. The number and size of HCC nodules influence treatment decisions, and access to liver transplantation list $[1,2]$. Thus, stringent criteria for a non-invasive diagnosis of $\mathrm{HCC}$ are mandatory [1,2]. The American Association for the Study of Liver Disease (ASSLD) 2010 guidelines recommend that a non-invasive diagnosis of $\mathrm{HCC}$ in patients with chronic

Francesco Agnello

fra.agnello@libero.it

1 Department of Radiology, University of Palermo, Via XII Gennaio 1/g, 90141 Palermo, Italy

2 Department of Sciences for Health Promotion and Mother and Child Care "G. D'Alessandro", University of Palermo, Palermo, Italy liver disease can be done at contrast-enhanced computed tomography (CT) or magnetic resonance imaging (MRI) if a nodule larger than $1 \mathrm{~cm}$ in diameter shows enhancement on hepatic arterial phase with subsequent wash-out on portal venous and/or delayed phase [1]. Using these criteria, MRI specificity is approximately $90 \%$ for $1-1.9 \mathrm{~cm} \mathrm{HCCs}$ and approximately $100 \%$ for $>2$-cm HCCs [3, 4]. These differences are related to vascular tumor changes during hepatocarcinogenesis, which result in an initial decrease in normal portal and arterial supply, subsequently followed by an increase in abnormal arterial supply via newly formed, abnormal, arteries [5]. Hepatocellular nodules without typical imaging features of HCC are considered indeterminate by ASSLD 2010 guidelines, and a second study (the other of CT or MRI) or nodule biopsy is recommended [1]. With the advent of Gd-EOB-DTPA, the ability of MRI to detect HCC nodules has increased, but in parallel, a growing number of hepatocellular nodules with indeterminate characteristics 
are being detected in the cirrhotic liver [6-8]. Most indeterminate hepatocellular nodules represent regenerative or dysplastic nodules, but a diagnosis of HCC cannot be ruled-out in almost all cases. In a radio-pathologic correlation study including 111 indeterminate hepatocellular nodules on Gd-EOB-DTPA MRI, Golfieri et al. have found 60 benign lesions, 41 premalignant lesions (high-grade dysplastic nodules/early HCC), and $10 \mathrm{HCCs}$, and concluded that hepatobiliary phase hypointensity is the stronger marker of malignancy [9]. The latter observation is in accordance with a paper by Kumada et al., which have reported that approximately one quarter of Gd-EOB-DTPA hepatobiliary phase hypointense hepatocellular nodules became hypervascular HCCs at follow-up [10]. Moreover, Kim et al. have found that hyperintensity on diffusion-weighted (DW) images in hypovascular hepatobiliary phase hypointense nodules increased the risk of progression to hypervascular HCCs [11]. However, these and similar studies have focused on nodules showing hypointensity on hepatobiliary phase and on their risk of HCC transformation. Other nodule imaging characteristics have not been extensively evaluated. To the best of our knowledge, there is only one study, which had specifically investigated the significance of hyperintense nodules on T1-weighted images [12].

Thus, the purpose of this study was to retrospectively analyze the evolution of indeterminate hepatocellular nodules of any type on serial Gd-EOB-DPTA-enhanced MRI in cirrhotic patients, and to identify nodule characteristics that allow prediction of HCC development.

\section{Materials and methods}

\section{Study population}

This retrospective study was performed in a large, referral hospital for hepatobiliary diseases (Policlinico Paolo Giaccone, University of Palermo). Institution review board of our hospital approved this study, and waived the need for patient informed consent because of the retrospective nature of the study.

The study coordinator (F.A., a radiology fellow with 8 years of experience) searched our radiology database to identify all cirrhotic patients who underwent GdEOB-DPTA-enhanced MRI of the liver between January 2009 and April 2015. To be included in this study, patients were required to have at least one hepatocellular nodule with indeterminate characteristics on cirrhotic liver at baseline Gd-EOB-DTPA-enhanced MRI, a mean baseline diameter of at least $5 \mathrm{~mm}$. Indeterminate hepatocellular nodules were defined as those nodules that did not fulfill the non-invasive diagnostic criteria for HCC diagnosis according to the ASSLD 2010 guidelines.

\section{A total of 98 patients were identified}

Of these, 55 were excluded for the following reasons: no Gd-EOB-DTPA MRI follow-up ( $n=25)$, Gd-EOB-DTPA MRI follow-up of less than 24 months $(n=10)$, any sort of treatment of the indeterminate hepatocellular nodule $(n=12)$, indeterminate hepatocellular nodule measuring less than $5 \mathrm{~mm}$ in diameter $(n=15)$, cholangiocarcinoma $(n=2)$, and motion artifacts, which made the examinations non-diagnostic $(n=1)$. Of the 10 who had less than 24-month follow-up, no nodule become hypervascular $\mathrm{HCC}$ at at multi-detector row CT or MRI follow-up or was proved to be HCC at pathology. In the presence of multiple hepatocellular indeterminate nodules, a maximum of three was selected per patient. Thus, the final study population was composed by 33 consecutive patients (mean age 64.9 years; range $45-76$ years) with 69 indeterminate hepatocellular nodules. Twenty-four were males (mean age 65.7 years; range 45-72) and 9 were females (mean age 63 , years; range 47-76). Fourteen patients had one nodule, 7 patients had 2 nodules, and 13 patients had three nodules. The etiology of cirrhosis was hepatitis $\mathrm{C}$ infection in 27 patients (82\%), hepatitis B infection in three patients (10\%), and both in three patients (10\%). The diagnosis of cirrhosis was made on the basis of histological findings in eight patients and combined clinical features and laboratory results in 25 patients.

\section{MR imaging protocol}

MR imaging was performed on one of the two 1.5 T MR scanners available at our institution (Signa Excite, General Electric, Healthcare; Intera Achieva, Philips Healthcare) equipped with a body-phased array coil. All MR images were acquired using parallel imaging sensitivity encoding with a reduction factor of two. The imaging protocol for both scanners included axial T2-weighted turbo or fast spin-echo (with and without fat saturation) sequences, axial dual gradient-recalled echo (GRE) T1-weighted sequence, axial diffusion-weighted (DW) sequence using three $b$ values $\left(0,150\right.$, and $\left.800 \mathrm{~s} / \mathrm{mm}^{2}\right)$, and axial contrastenhanced three-dimensional T1-weighted GRE sequence with fat suppression. A bolus of $0.25 \mathrm{mmol} / \mathrm{kg}$ of GDEOB-DTPA (Primovist, Bayer, Berlin, Germany) was injected at a rate of $1,1.5$, or $2 \mathrm{~mL} / \mathrm{s}$, followed by $20-\mathrm{mL}$ saline flush at the same injection rate, using an automated injector (Spectris; Medrad, Pittsburgh, PA, USA). Contrast-enhanced images are obtained immediately before and after contrast injection, on late hepatic arterial phase at 20-35 s, portal-venous phase at $80 \mathrm{~s}, 3-\mathrm{min}$ late, and 20-min hepatobiliary phases. An automated bolus 
detection algorithm (MR Smart Prep; GE Medical Systems, Milwaukee, WI, USA) or a fluoroscopic triggering (Bolus-Trak; Philips Medical Systems, Best, The Netherlands) was used to obtain an optimal hepatic arterial phase [13]. T2-weighted and DW sequences were obtained between the 3-min late and hepatobiliary phase to reduce the total examination time $[14,15]$. Other MRI parameters are shown in Table 1.

\section{Imaging analysis}

All MR images were independently analyzed on a picture archiving and communication system (PACS) (Impax, Agfa-Gevaert, Mortsel, Belgium) by two radiologists (D.A and M.G., with 5 and 15 years of experience in liver imaging, respectively). Discordance was resolved by consensus. Reviewers were informed that all patients were cirrhotic, but were blinded to all other patients' clinical data. When multiple follow-up MRI examinations were available, only the latest one was evaluated. The size and signal intensity of each nodule were evaluated at baseline and follow-up MRI.

Nodule size was measured as the longest cross-sectional diameter. Change in size was considered significant if there was a minimum increase or decrease in nodule diameter of $5 \mathrm{~mm}$ [16]. Nodule signal intensity was evaluated on unenhanced T1- and T2-weighted sequences, as well as on high- $b$-value DW and contrast-enhanced sequences, and was classified as hypointense, isointense, or hyperintense to the surrounding liver. On the basis of signal intensity at baseline MRI, each nodule was classified into one of the following six groups (hereafter called baseline groups): 1 , hypointense on hepatobiliary phase; 2 , hyperintense on hepatobiliary phase; 3 , hypointense on 3-min late and hepatobiliary phase; 4, hyperintense on T1-weighted and hypointense on hepatobiliary phase; 5 , hypointense on portal-venous, 3 -min late and hepatobiliary phase; and 6, hyperintense on T1-weighted phase.

The coexistence of other (at least three) nodules with the same imaging appearance of the selected nodule at baseline MRI was recorded.

Diagnosis of HCC was done according to the ASSLD 2010 guidelines criteria that require a minimum size of $10 \mathrm{~mm}$, and a typical enhancement pattern (i.e., arterial enhancement followed by venous wash-out) [1].

Medical charts were reviewed to determine whether any HCC exceeded the AASLD 2010 criteria for curative treatment, which include a single HCC more than $5 \mathrm{~cm}$ in diameter or $\leq 3 \mathrm{HCCs}$ less than $3 \mathrm{~cm} \mathrm{[1].}$

\section{Statistical analysis}

Statistical analysis was performed at per nodule level. The dependency among nodules observed within the same patient was explicitly considered in the analysis.

Data were compared between nodules that became HCCs at follow-up (hereafter called HCC nodules) and those that did not (hereafter called no-HCC nodules) using the Fisher's exact test for categorical variables and the generalized estimating equations model (GEE), with binomial family and logit link, for continuous variables. Age, cirrhosis etiology, and history of $\mathrm{HCC}$ before the baseline MR examination were also considered as a variable.

Data were reported as count and percentage for qualitative variables and as mean and standard deviation for continuous variables. A $p$ value of $<0.05$ was considered to indicate a statistically significant difference.

\begin{tabular}{lllll}
\hline Sequence & T2-w TSE & Dual T1-w GRE & DWI & 3D T1-w GRE \\
\hline $\begin{array}{llll}\text { 1.5 T Signa GE scanner } \\
\text { TR/TE (ms) }\end{array}$ & $4000 / 76$ & $140 / 2.2-4.4$ & $1600 / 72$ & $3.8 / 1.2$ \\
Flip angle $\left(^{\circ}\right)$ & 150 & 70 & 90 & 12 \\
Field of view (cm) & $35-40 \times 35-40$ & $35-40 \times 35-40$ & $35-40 \times 35-40$ & $35-40 \times 35-40$ \\
Matrix & $256 \times 224$ & $256 \times 160$ & $144 \times 192$ & $256 \times 256$ \\
Section thickness (mm) & 6 & 6 & 7 & 3 \\
Intersection gap (mm) & - & - & 1 & - \\
1.5 T Intera Philips scanner & & & & \\
TR/TE (ms) & $2500-8000 / 90$ & $236 / 2-4.3$ & $3448 / 64$ & $4.5 / 2.2$ \\
Flip angle $\left(^{\circ}\right)$ & 90 & 80 & 90 & 10 \\
Field of view (cm) & $35-40 \times 35-40$ & $35-40 \times 35-40$ & $35-40 \times 35-40$ & $35-40 \times 35-40$ \\
Matrix & $320 \times 320$ & $240 \times 256$ & $256 \times 120$ & $208 \times 256$ \\
Section thickness (mm) & 4 & 5 & 5 & 3 \\
Intersection gap (mm) & 1 & 1 & 1 & - \\
\hline
\end{tabular}

$W$ weighted, FSE fast spin echo, GRE gradient-recalled echo, $D W I$ diffusion-weighted imaging, $3 D$ threedimensional, TSE turbo spin echo 
Multivariate GEE model including all significant variables at the univariate analysis was used to identify independent predictors of HCC development. The adjusted odds ratios (ORs) with respective 95\% confidence intervals (CIs), sensitivity, specificity, positive predictive value (PPV), and negative predictive value (NPV) were reported. Statistical analysis was performed using a commercial statistical package software (Stata SE, versions 14.1; Stata, College Station, TX, USA).

\section{Results}

\section{Study population}

Of sixty-nine indeterminate hepatocellular nodules, only 5 (7\%) in five patients become hypervascular HCCs at followup (Fig. 1). The remaining 64 of 69 (93\%) nodules showed indeterminate characteristics (56 of $63,87 \%$ ), or disappeared $(8$ of $63,13 \%)$ at follow-up (Fig. 2$)$. The mean MRI follow-up was 805 days (range 785-2440 days). The mean time between the baseline MRI and HCC development was 551 days (range 413-735). No-HCC exceeded the AASLD 2010 criteria for curative treatment [1].

A history of HCC before the baseline MR examination was more frequently found in HCC nodules (4 of 5, 80\%) than in no-HCC nodules ( 29 of $64,45 \%)(p<0.005)$.

There were no statistically significant differences in the age, sex, and etiology of cirrhosis between the HCC and no-HCC nodules.

Demographic and clinical data are summarized in Table 2.

\section{Size}

The mean nodule size was $1.1 \mathrm{~cm}$ (range $0.5-2 \mathrm{~cm}$ ) at baseline, and $1.2 \mathrm{~cm}$ (range 0-6.6 cm) at follow-up, with a mean change in size of $0.27 \mathrm{~cm}$ (range $0.14-2.6 \mathrm{~cm}$ ). The HCC nodules had a greater mean baseline diameter than non-HCC nodules, and these differences were statistically significant.

The mean tumor diameter doubling time of HCC nodules was 595.25 days. Of the 64 no-HCC nodules, 12 of 64 (19\%) increased in size at follow-up, 37 of $64(58 \%)$ were stable in size, and 15 of 64 (23\%) decreased in size. Among 12 no-HCC nodules with increased size at follow-up, 10 of 12 $(83 \%)$ were high-grade dysplastic nodules and 2 of $12(17 \%)$ were low-grade dysplastic nodules.

Diagnosis of no-HCC was confirmed by means of surgical resection (two high-grade dysplastic nodules) and fineneedle biopsy (eight high-grade dysplastic nodules and two low-grade dysplastic nodules).

HCC nodules had a significantly greater mean change in size than no-HCC nodules. Details are shown in Table 3.
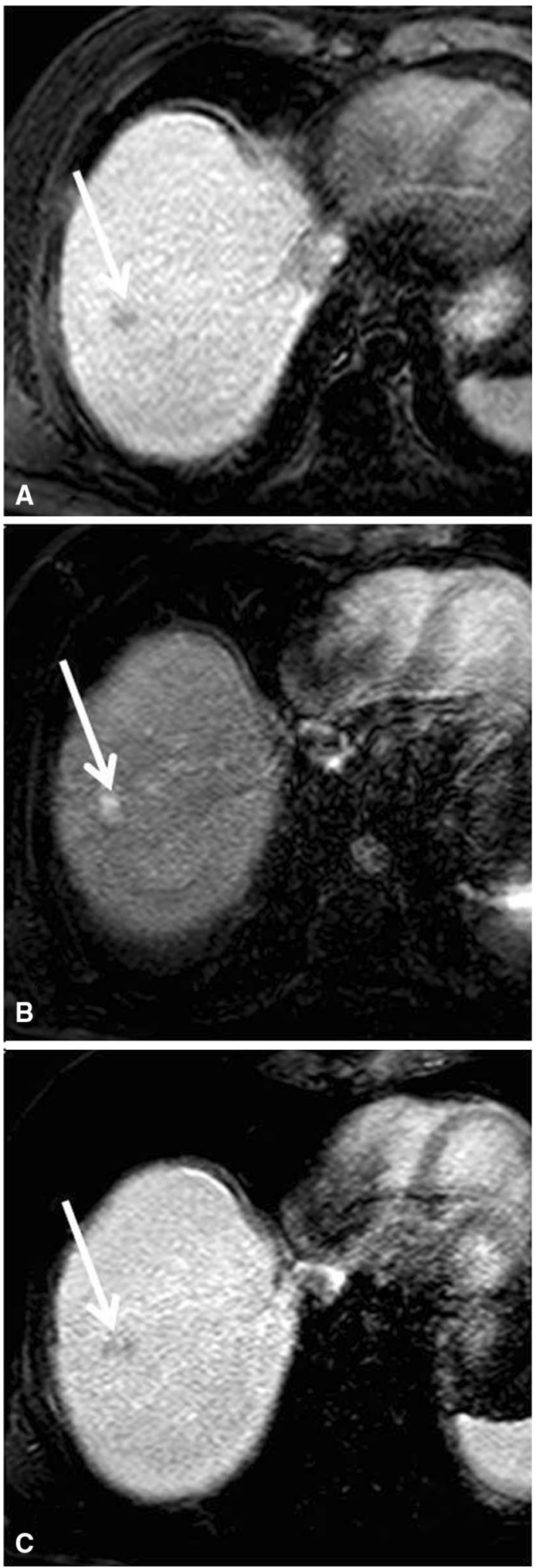

Fig. 1 HCC nodule in a 65-year-old man with HCV-related cirrhosis. a On baseline MRI, the nodule (arrow) shows hypointensity on hepatobiliary phase. b, c On 15-month MR follow-up, the nodule shows enhancement on hepatic arterial phase (b) and wash-out on portalvenous phase (b) 

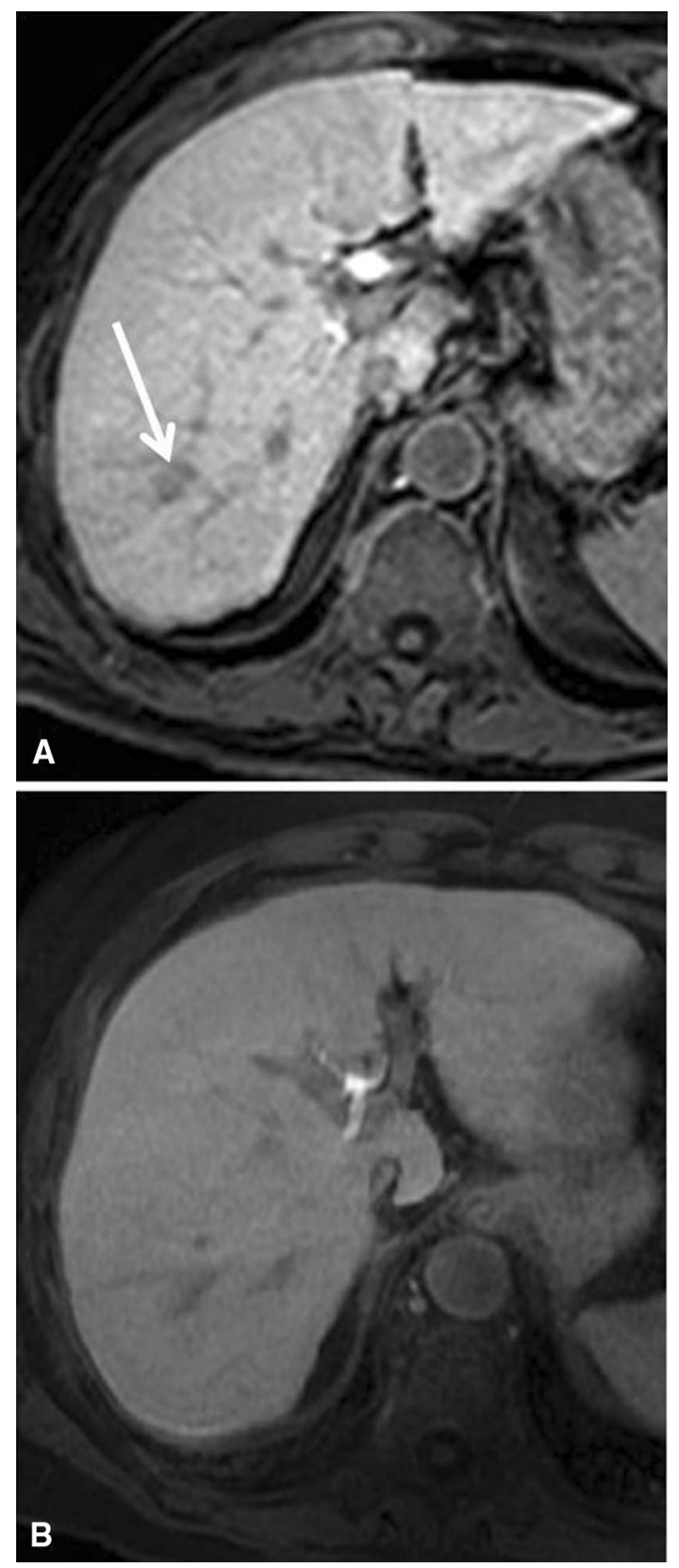

Fig. 2 No-HCC nodule in a 63-year-old man with HCV-related cirrhosis. a Baseline hepatobiliary phase MR image shows a hypointense nodule in the right hepatic lobe (arrow). b On 18-month hepatobiliary phase MR image, the nodule is not appreciable

\section{Signal intensity characteristics}

Hypointensity on hepatobiliary phase was significantly more common in HCC than in no-HCC nodules. Other baseline signal intensity characteristics of HCC nodules were not significantly different from that of no-HCC nodules. However, there were trends toward HCC development, which did not reach statistical significance among nodules that showed hypointensity on 3-min late phase. Details are shown in Table 3.

\section{Multivariate analysis}

Multivariate regression analysis included history of HCC, baseline size, increase in size, and hypointensity on hepatobiliary phase. We found that increase in size [OR 10.48 (95 CIs 2.99-36.74); sensitivity, 100\%; specificity, 81.2\%; PPV, $31.3 \%$, NPV, $100 \%$; $p<0.001$ ], and hypointensity on hepatobiliary phase [OR 1.02 (95 CIs 0.21-5.05); sensitivity, $100 \%$; specificity, $78.1 \%$; PPV, $16.7 \%$; NPV, $100 \%$; $p=<0.001$ ], were associated with HCC development. Other variables failed to achieve a statistical significance.

\section{Discussion}

In this study, we have analyzed the evolution of indeterminate hepatocellular nodules in cirrhotic patients in serial GdEOB-DPTA-enhanced MRI. We found that only few nodules became hypervascular HCCs at follow-up, and the majority of nodules showed indeterminate findings or disappeared at follow-up.

The frequency of HCC transformation of indeterminate hepatocellular nodules was in line with the previous results [17-19]. In a study using contrast-enhanced CT, Chung et al. have reported that 15 of 634 (2.4\%) non-hypervascular, hypoattenuating, and hepatocellular nodules transformed into hypervascular HCCs on serial imaging follow-up [17]. Differences between our results and those by Chung et al. are probably related to the higher sensitivity of MRI compared to CT in the diagnosis of HCC [20]. The observation that indeterminate hepatocellular nodules rarely become hypervascular HCCs is also supported by a study by Seki et al., which have reported that most dysplastic nodules disappeared (15 of 33, 45\%) or remained unchanged (14 of $33,42 \%)$ at follow-up [18]. The fact that dysplastic nodules rarely progress into HCCs has also been described by Farber et al. in a study using a rat model [19].

The HCC nodules had a slow grow on a serial imaging follow-up, suggesting that a 6-12-month follow-up is the best option for the management of indeterminate hepatocellular nodules detected at Gd-EOB-DTPA-enhanced MRI. Nodule biopsy may represent an alternative option, but the invasiveness and risk of complications (e.g., bleeding) strongly limit its usage [21]. Of note, in our series, all noHCCs with increased size at follow-up were high- or lowgrade dysplastic nodules at pathology analysis.

Since the goal of screening cirrhotic patients is to detect HCC at an early stage, the second purpose of our study was to identify nodule characteristics that can predict HCC development $[1,22,23]$. We found that the only 
Table 2 Demographic and clinical features

\begin{tabular}{|c|c|c|c|c|}
\hline \multirow[t]{3}{*}{ Parameter } & \multicolumn{4}{|c|}{ Final diagnosis } \\
\hline & \multirow[t]{2}{*}{$\operatorname{HCC}(N=5)$} & \multicolumn{3}{|l|}{ No-HCC } \\
\hline & & Total $(N=64)$ & Group A $(N=56)$ & Group B $(N=8)$ \\
\hline $\mathrm{Age}^{\mathrm{a}}$ & $67(56-78)$ & $64.4(41-82)$ & $65.9(41-82)$ & $54.4(44-63)$ \\
\hline \multicolumn{5}{|l|}{ Sex } \\
\hline Male & $4(80)$ & $54(84)$ & $47(84)$ & $7(87)$ \\
\hline Female & $1(20)$ & $10(16)$ & $9(16)$ & 1(13) \\
\hline \multicolumn{5}{|l|}{ Cirrhosis etiology } \\
\hline Hepatitis $\mathrm{C}$ virus & $5(100)$ & $48(75)$ & $40(72)$ & $8(100)$ \\
\hline Hepatitis B virus & - & $13(20)$ & $13(23)$ & - \\
\hline Hepatitis $\mathrm{C}$ and $\mathrm{B}$ virus & - & $3(5)$ & $3(5)$ & - \\
\hline \multicolumn{5}{|l|}{ History of HCC } \\
\hline Yes & $4(80.0)$ & $29(45)$ & $26(46)$ & $3(37)$ \\
\hline No & $1(20.0)$ & $35(55)$ & $30(54)$ & $5(63)$ \\
\hline
\end{tabular}

Group A included nodules that showed indeterminate characteristics at follow-up. Group B included nodules that disappeared at follow-up. Unless otherwise indicated, data are numbers. Data in parentheses are percentages. Data in parentheses are ranges

${ }^{a}$ Data are mean \pm standard deviation baseline independent predictor of HCC development was the hepatobiliary phase hypointensity. Due to the observation of this feature in all HCCs, its application as independent predictor of HCC development had a sensitivity of $100 \%$. Hepatobiliary phase hypointensity was attributed to decreased organic anion-transporting polypeptide (OATP8) expression, which occurs during the multistep hepatocarcinogenesis in parallel with elevation of nodule malignancy grade, before the reduction of normal arterioportal inflow and elevation of abnormal arterial inflow [24]. This allowed us to speculate that hepatobiliary phase hypointense nodules, which subsequently became HCCs, might be high-grade dysplastic nodules or early HCCs [25, 26]. In a study including sixty-six consecutive cirrhotic patients with seventy pathologically proved hepatocellular nodules, Lee et al. reported that well-differentiated HCCs showed more frequently hepatobiliary phase hypointensity than benign hepatocellular nodules [27]. However, in our series, hepatobiliary phase hypointensity had a specificity of $78 \%$ and a very week OR (1.2), because most hepatocellular nodules showing hepatobiliary phase hypointensity remained indeterminate, or disappeared at follow-up. In view of this, hepatobiliary phase hypointensity might not necessarily suggest potential HCC transformation, and its application as a predictor of HCC development should be undertaken cautiously. This is supported by the fact that hepatobiliary phase hypointensity was considered by the Liver Imaging Reporting and Data System (LIRADS) as an ancillary feature that favors the diagnosis of HCC and not a major feature of HCC [28]. The fact that the hepatobiliary phase hypointensity can be found in any non-hepatocyte containing lesion (e.g., cysts and hemangiomas) further limits its application as an independent predictor for HCC development [28-30]. No-HCC nodules showing hepatobiliary phase hypointensity are likely regenerative or dysplastic nodules.

Another interesting result of our study was that, although HCC nodules had a greater mean baseline diameter than noHCC nodules, the baseline size was not a predictive factor for HCC development.

On the other hand, nodule increase in size significantly correlated with HCC transformation, and represented an independent predictor of HCC development. In our series, an increase in size was observed in all HCC- nodules and in few no-HCC nodules. Thus, it is crucial to pay particular attention and carefully observe those hepatocellular nodules, which show hepatobiliary phase hypointensity and increase in size.

This study had some limitations that should be pointed out. First, the number of indeterminate hepatocellular nodules was relatively small. However, this reflects our clinical practice, in which imaging follow-up is performed with different modalities and suspicious nodules are treated as soon as possible to prevent the development of untreatable HCCs. Second, pathologic analysis of all nodules was not performed. Therefore, we cannot exclude that some indeterminate hepatocellular nodules may be atypical HCCs. However, the probability that a no-HCC nodule with stable or decreased size at follow-up was an HCC was extremely low because of a long follow-up [31, 32]. Finally, there was no a control group of HCC nodules without indeterminate findings at the previous Gd-EOB-DTPA-enhanced MR studies. Comparison of HCCs with and without indeterminate findings at the previous Gd-EOB-DTPA-enhanced MR studies 
Table 3 Imaging features

\begin{tabular}{|c|c|c|c|c|}
\hline \multirow[t]{3}{*}{ Parameter } & \multicolumn{4}{|l|}{ Final diagnosis } \\
\hline & \multirow[t]{2}{*}{$\operatorname{HCC}(N=5)$} & \multicolumn{3}{|l|}{ No-HCC } \\
\hline & & Total $(N=64)$ & Group A $(N=56)$ & Group B $(N=8)$ \\
\hline Initial size $(\mathrm{mm})^{\mathrm{a}}$ & $20.4 \pm 11.19(12 ; 40)$ & $10.95 \pm 5.04(4 ; 30)$ & $11.43 \pm 5.06(4 ; 30)$ & $7.6 \pm 3.54(4 ; 14)$ \\
\hline Change in size $(\mathrm{mm})^{\mathrm{a}}$ & $10 \pm 9.03(5 ; 26)$ & $-0.48 \pm 3.99(-14 ; 12)$ & $0.54 \pm 2.8(-5 ; 12)$ & $-7.63 \pm 3.54(-14 ;-4)$ \\
\hline \multicolumn{5}{|l|}{ Change in size } \\
\hline Increased & $5(100)$ & $12(19)$ & $12(21)$ & - \\
\hline Decreased & - & $15(23)$ & $7(12)$ & $8(100)$ \\
\hline Stable & - & $37(58)$ & $37(66)$ & - \\
\hline \multicolumn{5}{|l|}{ SI on T2-w phase } \\
\hline Hypointense & - & - & - & - \\
\hline Isointense & $5(100)$ & $64(100)$ & $56(100)$ & $8(100)$ \\
\hline Hyperintense & - & - & - & - \\
\hline \multicolumn{5}{|l|}{ SI on T2-w fat-sat phase } \\
\hline Hypointense & - & - & - & - \\
\hline Isointense & $5(100)$ & $64(100)$ & $56(100)$ & $8(100)$ \\
\hline Hyperintense & - & - & - & - \\
\hline \multicolumn{5}{|c|}{ SI on high-b-value DW phase } \\
\hline Hypointense & - & - & - & - \\
\hline Isointense & $5(100)$ & $64(100)$ & $56(100)$ & $8(100)$ \\
\hline Hyperintense & - & - & - & - \\
\hline \multicolumn{5}{|l|}{ SI on T1-w phase } \\
\hline Hypointense & - & $3(5)$ & $3(5)$ & - \\
\hline Isointense & $4(80)$ & $45(70)$ & $38(68)$ & $7(87)$ \\
\hline Hyperintense & $1(20)$ & $16(25)$ & $15(27)$ & $1(13)$ \\
\hline \multicolumn{5}{|l|}{ SI on HAP phase } \\
\hline Hypointense & - & $3(5)$ & $3(5)$ & - \\
\hline Isointense & $5(100)$ & $59(92)$ & $51(91)$ & $8(100)$ \\
\hline Hyperintense & - & $2(3)$ & $2(4)$ & - \\
\hline \multicolumn{5}{|l|}{ SI on PVP phase } \\
\hline Hypointense & - & $9(14)$ & $8(14)$ & $1(13)$ \\
\hline Isointense & $5(100)$ & $55(86)$ & $48(86)$ & $7(87)$ \\
\hline Hyperintense & - & - & - & - \\
\hline \multicolumn{5}{|l|}{ SI on 3-min late phase } \\
\hline Hypointense & $2(40)$ & $13(20)$ & $12(21)$ & $1(13)$ \\
\hline Isointense & $3(60)$ & $49(77)$ & $42(75)$ & $7(87)$ \\
\hline Hyperintense & - & $2(3)$ & $2(4)$ & - \\
\hline \multicolumn{5}{|l|}{ SI on 20-min HB phase } \\
\hline Hypointense & $5(100)$ & $25(39)$ & $25(45)$ & - \\
\hline Isointense & - & $15(24)$ & $12(21)$ & $3(37)$ \\
\hline Hyperintense & - & 24 (37) & $19(34)$ & $5(63)$ \\
\hline \multicolumn{5}{|l|}{ Baseline group } \\
\hline 1 & $2(40)$ & $7(11)$ & $7(12)$ & - \\
\hline 2 & - & $23(36)$ & $18(32)$ & $5(63)$ \\
\hline 3 & $3(60)$ & 17 (26.5) & $15(27)$ & $2(25)$ \\
\hline 4 & - & $4(6)$ & $4(7)$ & - \\
\hline 5 & - & $1(1.5)$ & $1(2)$ & - \\
\hline 6 & - & $12(19)$ & $11(20)$ & $1(12)$ \\
\hline \multicolumn{5}{|l|}{ Other nodules } \\
\hline Yes & $4(80.0)$ & $27(42)$ & $22(39)$ & $5(63)$ \\
\hline
\end{tabular}


Table 3 (continued)

\begin{tabular}{lllll}
\hline & \multicolumn{2}{l}{ Final diagnosis } & & \\
\cline { 2 - 5 } & & NCC $(N=5)$ & Group A $(N=56)$ & Group B $(N=8)$ \\
\cline { 2 - 5 } & $1(20.0)$ & Total $(N=64)$ & $34(6)$ & $3(37)$ \\
\hline No & $37(58)$ & & \\
\hline
\end{tabular}

Group A included nodules that showed indeterminate characteristics at follow-up. Group B included nodules that disappeared at follow-up. Baseline group $=$ group of nodules at baseline MRI

Unless otherwise indicated, data are numbers. Data in parentheses are percentages. Data in parentheses are ranges

$S I$ signal intensity, Other nodules other nodules with the same imaging appearance of the selected nodule at initial MRI, $W$ weighted, $D W$ diffusion weighted, $H A P$ hepatic arterial phase. $P V P$ portal-venous phase, $H B$ hepatobiliary phase

${ }^{a}$ Data are mean \pm standard deviation

could help to identify those patients at higher risk for HCC development.

In conclusion, this study demonstrated that indeterminate hepatocellular nodules in cirrhotic patients rarely progress into hypervascular HCCs. Nodules with baseline hepatobiliary phase hypointensity and increase in size at followup might be prone to become hypervascular HCCs. A GdEOB-DTPA-enhanced MRI follow-up could represent the best option for the management of indeterminate hepatocellular nodules on Gd-EOB-DTPA-enhanced MRI.

Funding This study did not receive any funding.

\section{Compliance with ethical standards}

Conflict of interest Authors declare that they have no conflict of interest.

Ethical standards This article does not contain any studies with human participants or animals performed by any of the authors.

\section{References}

1. Bruix J, Sherman M, American Association for the Study of Liver Diseases (2011) Management of hepatocellular carcinoma: an update. Hepatology 53(3):1020-1022

2. HRSA/OPTN. Policy 3.6 organ distribution: allocation of livers. 2012. http://optn.transplant.hrsa.gov/policiesAndBylaws/policies. asp. Accessed Jan 2017

3. Marrero JA, Hussain HK, Nghiem HV, Umar R, Fontana RJ, Lok AS (2005) Improving the prediction of hepatocellular carcinoma in cirrhotic patients with an arterially-enhancing liver mass. Liver Transpl 11(3):281-289

4. Forner A, Vilana R, Ayuso C et al (2008) Diagnosis of hepatic nodules $20 \mathrm{~mm}$ or smaller in cirrhosis: prospective validation of the noninvasive diagnostic criteria for hepatocellular carcinoma. Hepatology 47(1):97-104

5. Matsui O (2004) Imaging of multistep human hepatocarcinogenesis by CT during intra-arterial contrast injection. Intervirology 47(3-5):271-276

6. Ahn SS, Kim MJ, Lim JS, Hong HS, Chung YE, Choi JY (2010) Added value of gadoxetic acid-enhanced hepatobiliary phase MR imaging in the diagnosis of hepatocellular carcinoma. Radiology 255(2):459-466

7. Golfieri R, Renzulli M, Lucidi V, Corcioni B, Trevisani F, Bolondi L (2011) Contribution of the hepatobiliary phase of Gd-EOB-DTPA-enhanced MRI to Dynamic MRI in the detection of hypovascular small $(\leq 2 \mathrm{~cm}) \mathrm{HCC}$ in cirrhosis. Eur Radiol 21(6): 1233-1242

8. Haradome H, Grazioli L, Tinti R et al (2011) Additional value of gadoxetic acid-DTPA-enhanced hepatobiliary phase MR imaging in the diagnosis of early-stage hepatocellular carcinoma: comparison with dynamic triple-phase multidetector CT imaging. J Magn Reson Imaging 34(1):69-78

9. Golfieri R, Grazioli L, Orlando E et al (2012) Which is the best MRI marker of malignancy for atypical cirrhotic nodules: hypointensity in hepatobiliary phase alone or combined with other features? Classification after Gd-EOB-DTPA administration. J Magn Reson Imaging 36(3):648-657

10. Kumada T, Toyoda H, Tada T et al (2011) Evolution of hypointense hepatocellular nodules observed only in the hepatobiliary phase of gadoxetate disodium-enhanced MRI. AJR 197(1):58-63

11. Kim TK, Lee KH, Jang HJ et al (2011) Analysis of gadobenate dimeglumine-enhanced MR findings for characterizing small $(1-2-\mathrm{cm})$ hepatic nodules in patients at high risk for hepatocellular carcinoma. Radiology 259(3):730-738

12. Suh YJ, Kim MJ, Choi JY, Park YN, Park MS, Kim KW (2011) Differentiation of hepatic hyperintense lesions seen on gadoxetic acid-enhanced hepatobiliary phase MRI. AJR 197(1):W44-W52

13. Tanimoto A, Lee JM, Murakami T, Huppertz A, Kudo M, Grazioli L (2009) Consensus report of the 2nd international forum for liver MRI. Eur Radiol 19(Suppl 5):S975-S989

14. Tamada T, Ito K, Yoshida K et al (2010) T2-weighted magnetic resonance imaging of the liver: evaluation of the effect in signal intensity after Gd-EOB-DTPA enhancement. J Comput Assist Tomogr 34:182-186

15. Muhi A, Ichikawa T, Motosugi U, Sou H, Sano K, Araki T (2012) Diffusion- and T2-weighted MR imaging of the liver: effect of intravenous administration of gadoxetic acid disodium. Magn Reson Med Sci. 11:185-191

16. Eisenhauer EA, Therasse P, Bogaerts J et al (2009) New response evaluation criteria in solid tumours: revised RECIST guideline (version 1.1). Eur J Cancer 45:228-247

17. Chung JJ, Yu JS, Kim JH, Kim MJ, Kim KW (2008) Non hypervascular hypoattenuating nodules depicted on either portal or equilibrium phase multiphasic CT images in the cirrhotic liver. AJR 191(1):207-214

18. Seki S, Sakaguchi H, Kitada T et al (2000) Outcomes of dysplastic nodules in human cirrhotic liver: a clinicopathological study. Clin Cancer Res 6(9):3469-3473 
19. Farber E, Cameron R (1980) The sequential analysis of cancer development. Adv Cancer Res 31:125-226

20. Colli A, Fraquelli M, Casazza G et al (2006) Accuracy of ultrasonography, spiral CT, magnetic resonance, and alpha-fetoprotein in diagnosing hepatocellular carcinoma: a systematic review. Am J Gastroenterol 101(3):513-523

21. Heimbach J, Kulik LM, Finn R, Sirlin CB, Abecassis M, Roberts LR, Zhu A, Murad MH, Marrero J (2017) AASLD guidelines for the treatment of hepatocellular carcinoma. Hepatology 67(1):358-380

22. European Association for the Study of the Liver, European Organization For Research And Treatment Of Cancer (2012) EASLEORTC clinical practice guidelines: management of hepatocellular carcinoma. J Hepatol 56:908-943

23. Omata M, Lesmana LA, Tateishi R et al (2010) Asian Pacific Association for the Study of the Liver consensus recommendations on hepatocellular carcinoma. Hepatol Int 4:439-474

24. Kitao A, Zen Y, Matsui O et al (2010) Hepatocellular carcinoma: signal intensity at gadoxetic acid-enhanced MR Imaging-correlation with molecular transporters and histopathologic features. Radiology 256(3):817-826

25. Kim YK, Lee WJ, Park MJ, Kim SH, Rhim H, Choi D (2012) Hypovascular hypointense nodules on hepatobiliary phase gadoxetic acid-enhanced MR images in patients with cirrhosis: potential of DW imaging in predicting progression to hypervascular HCC. Radiology 265(1):104-114
26. Kudo M (2009) Multistep human hepatocarcinogenesis: correlation of imaging with pathology. J Gastroenterol 44(19):112-118

27. Lee MH, Kim SH, Park MJ, Park CK, Rhim H (2011) Gadoxetic acid-enhanced hepatobiliary phase MRI and high-b-value diffusion-weighted imaging to distinguish well-differentiated hepatocellular carcinomas from benign nodules in patients with chronic liver disease. AJR 197(5):W868-W875

28. American College of Radiology. Liver Imaging Reporting and Data System Version 2014. ACR Web site http://www.acr.org/ QualitySafety/Resources/LIRADS. Accessed Jan 2017

29. Tamada T, Ito K, Yamamoto A et al (2011) Hepatic hemangiomas: evaluation of enhancement patterns at dynamic MRI with gadoxetate disodium. AJR 196(4):824-830

30. Frydrychowicz A, Lubner MG, Brown JJ et al (2012) Hepatobiliary MR imaging with gadolinium-based contrast agents. J Magn Reson Imaging 35(3):492-511

31. Ebara M, Ohto M, Shinagawa T et al (1986) Natural history of minute hepatocellular carcinoma smaller than three centimeters complicating cirrhosis. A study in 22 patients. Gastroenterology 90(2):289-298

32. Barbara L, Benzi G, Gaiani S et al (1992) Natural history of small untreated hepatocellular carcinoma in cirrhosis: a multivariate analysis of prognostic factors of tumor growth rate and patient survival. Hepatology 16(1):132-137 